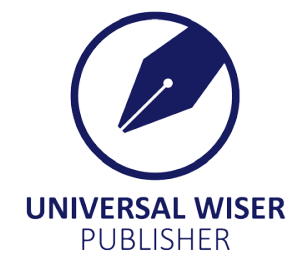

\title{
Modelling and Parameter Extraction of PV Cell Using Single-Diode Model
}

\author{
Mohammed Rasheed", Suha Shihab \\ Applied Sciences Department, University of Technology, Baghdad, Iraq \\ Email: rasheed.mohammed40@yahoo.com, 10606@uotechnology.edu.iq
}

\begin{abstract}
In this work, numerical solution of nonlinear equations using Newton Raphson method (NRM) and a modified Newton-Raphson Method (MNRM) are utilized to solve and find the real roots of a nonlinear equation based on a single-diode PV cell. The proposed methods to solve nonlinear examples and obtain results with various values of a load resistance have been examined. The purpose of this paper is to obtain the results of solar cell parameters using two mathematical models with the comparison between them. The obtained results showed the proposed method (MNRM) is a powerful tool, sufficient way to solve this model with a least iterations.
\end{abstract}

Keywords: single-diode model, Newton-Raphson method, solar cell, numerical analysis, modified Newton-Raphson method, nonlinear equation

\section{Introduction}

Many numerical analysis and approximation techniques have been suggested with some conditions to solve nonlinear equations $g(x)=0$. These methods are demonstrated based on different techniques. There are many definitions of numerical analysis can be summarized, included in one comprehensive definition, and most important: First is the choice of a method for finding approximate solutions to a numerical problem. Second is finding the equation (function) of numerical data against special conditions. Numerical analysis involves the study and evaluation of methods for calculating targeted numerical results for simple numerical data. It is part of modern science known as numerical information processing. The information (data) is referred to as "Input Information", the calculation method as "Algorithm", and the target results as "Output Information". Numerical analysis is a branch of mathematics used in many fields, for example, authors in [1] studied state parameterization for solving optimal control problem. Author in [2] treated modified basic spline multiscaling and wavelets algorithms for optimal control problems. While operational matrix basic spline wavelets of derivative for linear optimal control problem has been studied in [3]. In addition, modified iterative algorithm for solving optimal control problems have been used in [4]. Moreover, direct iterative algorithm for solving optimal control problems using B-spline polynomials has been investigated in [5]. Photon conversion to an electron the solar cells used in calculators and in satellites are photovoltaic cells, a group of electrical cells connected to each other in a single panel-shaped frame. Photovoltaic is a name derived from the nature of the cell's work. "Photo" means light, and "Voltec" means electricity, which means converting sunlight into electricity. Initially, photovoltaic cells were used in satellites and space stations to get electricity directly from the sun, and now they are entering many electronic devices and cars.

One can solve the IV curve of solar cell numerically depends on how the equation of IV curve model looks like the one-diode or two-diode model, with or without series and/or shunt resistance. Usually, to obtain a single point of the IV curve one has to solve an implicit equation, which can be reformulated as a zero-finding problem. This means that you have to perform the numerical solution repeatedly, i.e. in a loop (as many times as the number of desired amount of data points).

The parameters of the solar cells using different methods and numerical algorithms such as visual studio program ${ }^{[6]}$, fuzzy logic method $^{[7-8]}$, fuzzy set technique ${ }^{[9]}$; have been implemented and reported by Rasheed et al.

Other researchers can be use the numerical methods for solving nonlinear equation of different structures of solar cells based on the method of preparation and deals with inorganic and organic materials such as thin films solar cells, silicon solar cells, and ceramic materials ${ }^{[10]}$. 
The present work aim is to propose and describe a new method in order to find the real roots of single-diode nonlinear equation of the solar cells. It is organized as follows: section 2 presents characterizing of the analytical model of a singlediode design of the solar cell; Section 3 establishes the root using NRM and MNRM; while in section 4 results and discussion are included. Section 5 contains conclusions of the obtained results. Matlab program is used for all the obtained results.

\section{Characteristics of single-diode solar cells equation}

In electrical engineering and science, an equivalent circuit refers to a theoretical circuit that maintains all the electrical properties of a given circuit. The equivalent circuit is believed to simplify calculations, and on a larger scale, it is the simpler form of a more complex circuit to aid in it's analysis. In it's common form, the equivalent circuit is made up of linear and passive components, but more complex parabolic circuits approximate the nonlinear behavior of the original circuit as well. In order to understand the behavior of the solar cell, an equivalent electrical model had to be made. This model is based on known electrical components that are easy to study and analyze.

In the ideal model of solar cells, the cell is represented by a source of electric current with a uniform diode, but practically there is no ideal solar cell, so a resistance is placed in parallel, the shunt resistance, and another resistance respectively, as a simulation of reality. The simple an equivalent electric circuit of a solar cell is shown in Figure 1.

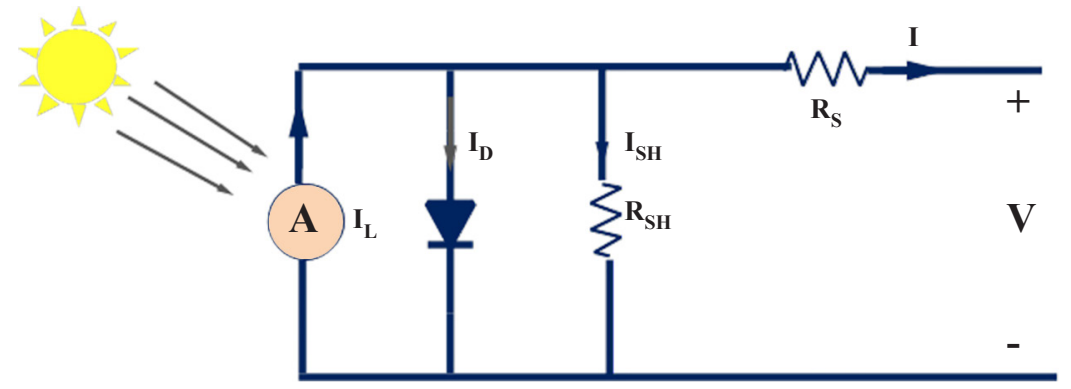

Figure 1. Equivalent-circuit of a single-diode model

Using Kiechoff's current law for the current $I$, the equation of this equivalent circuit is given by

The current from the solar cell = the current from the source IL - the current passing through the diode combiner ID the current passing through the resistor in the parallel $R_{S H}$

Voltage on both ends of the combiner diode $=$ Voltage on terminals $V+$ Current $\times$ Resistance (IR). Thus;

$$
\begin{aligned}
& I=I_{p h}-I_{D} \\
& I_{D}=I_{0}\left(e^{\frac{-V_{p v}}{n V_{T}}}-1\right) \\
& I=I_{p h}-I_{0}\left(e^{\frac{-V_{p v}}{m V_{T}}}-1\right)
\end{aligned}
$$

where:

$I_{p h}$ is the photocurrent $(A) ; I_{0}$ is reverse saturation current of the diode $(A) ; I$ and $V_{p v}$ are the delivered current and voltage, respectively $(V) ; V_{T}=\frac{k T}{q}=0.0259 \mathrm{~V}$ is thermic voltage $=27.5 \cong 26 \mathrm{mV}$ at $\left(T=25^{\circ} \mathrm{C}\right.$ Air-Mass $\left.=1.5\right)$; $m$ is the recombination factor closeness to an ideal diode $\left(1<m<2, k\right.$ is Boltzmann constant $=1.38 \times 10^{-23} \mathrm{~J} / \mathrm{K}$; $T$ is p-n junction temperature $(K) ; q$ is the electron charge $=1.6 \times 10^{-19} \mathrm{C}$.

$$
I_{\text {ph }}=I_{\text {source }}
$$




$$
I_{D}=I_{s} \times\left(e^{\frac{V_{0}}{n V_{T}}}-1\right)
$$

Merge Eq. 4 in Eq. 5 we get

$$
\left(I_{\text {source }}\right)-10^{-12}\left(e^{\frac{-V}{1.2 * 0.026}}-1\right)=\frac{V}{R}
$$

where $I_{s}$ reverse saturation current $=10^{-12} \mathrm{~A}$. In parallel, $V_{D}=V_{p v}=V$

According to Eq. 6 one can calculate $V$ of the cell numerically based on the first derivative of this equation.

\section{Mathematical methods}

\subsection{Newton-Raphson method (NRM)}

If we can isolate one root of the equation $f(x)=0$ in the period $[a, b]$ and suppose that $y=f(x)$ is a continuous function within this period. Then if we draw the graph curve of $y=f(x)$ then we take a point such as $x_{0}$ in the period $[a, b]$ and found $f\left(x_{0}\right)$ and draw the tangent of the function $y=f(x)$ at the point $\left(x_{0}, f\left(x_{0}\right)\right)$, where the equation of the tangent line passing from the point $\left(x_{0}, f\left(x_{0}\right)\right)$ and its slope $m=\dot{f}\left(x_{0}\right)$ is given by the following relation:

$$
y-y_{0}=f(x)-f\left(x_{0}\right)=m\left(x-x_{0}\right)=\dot{f}\left(x_{0}\right)\left(x-x_{0}\right)
$$

Assume that this tangent cuts the $x$-axis at a point $x_{1}$. Since $\left(x_{1}, 0\right)$ the point of intersection of this tangent with the axis of the $x$-axis, if we change all $x$ by $x_{1}$ and $y$ by 0 , we find

$$
0-f\left(x_{0}\right)=f^{\prime}\left(x_{0}\right)\left(x_{1}-x_{0}\right)
$$

then

$$
x_{1}-x_{0}=\frac{-f\left(x_{0}\right)}{\dot{f}\left(x_{0}\right)} \rightarrow x_{1}=x_{0}-\frac{f\left(x_{0}\right)}{\dot{f}\left(x_{0}\right)}
$$

Then we find the tangent of the function $y=f(x)$ at the point $\left(x_{1}, f\left(x_{1}\right)\right)$ and its slop $m=\dot{f}\left(x_{1}\right)$, the new tangent cuts the $x$-axis at a point $x_{2}$, so by writing the equation of that tangent passing from the point $\left(x_{1}, f\left(x_{1}\right)\right)$ and its tangent $m=\dot{f}\left(x_{1}\right)$ we find

$$
f(x)-f\left(x_{1}\right)=\dot{f}\left(x_{1}\right)\left(x-x_{1}\right)
$$

Since the intersection point $\left(x_{2}, 0\right)$ of this tangent with the $x$-axis, if we substitute each $x$ by $x_{2}$ and $y=f(x)=0$, we find

$$
0-f\left(x_{1}\right)=\dot{f}\left(x_{1}\right)\left(x_{2}-x_{1}\right)
$$

Then $x_{2}=x_{1}-\frac{f\left(x_{n}\right)}{f\left(x_{n}\right)}$ and by repeating this process a number of times, we get the following general formula

$$
x_{n+1}=x_{n}-\frac{f\left(x_{n}\right)}{\dot{f}\left(x_{n}\right)}, n=0,1,2, \ldots
$$

This formula is called the Newton-Raphson formula.

The point $x_{n+1}$ can be considered an approximate root of equation $f(x)=0$ if the condition is met 


$$
\left|x_{n+1}-x_{n}\right|<\varepsilon
$$

where $\varepsilon$ is a very small number.

\subsection{Modified Newton-Raphson method (MNRM)}

For a given $x_{0}$

$x_{n+1}=x_{n}-\frac{f\left(x_{n}\right)}{f\left(x_{n}\right)}$

Let $u(x) \frac{f\left(x_{n}\right)}{f\left(x_{n}\right)}, f(x)$ goes to zero before $f\left(x_{n}\right)$ does

$x_{n+1}=x_{n}+\frac{u(x)}{u(x)}$

$\dot{u}(x)=\frac{f(x) \times \dot{f}(x)-f(x) \times \dot{f}(x)}{[\dot{f}(x)]^{2}}$

Then

$x_{n+1}=x_{n}-\frac{f\left(x_{n}\right) \times \dot{f}\left(x_{n}\right)}{[\dot{f}(x)]^{2}-f\left(x_{n}\right) \times \dot{f}(x)}$

The above equation is called Modified Newton-Raphson Method.

\section{Results and discussion}

Consider Eq. 6 which is modeled in the form single-diode PV cell. It's approximate solutions is obtained and the two different methods are applied with the initial value $x_{0}=1$. Table 1 shows NRM and MNRM methods with the comparison of the results obtained are given and listed in the last columns of this table when the load resistance $R=1$. From this table, the result of the iterative process and the minimum stopping criterion $\varepsilon$, in which the objective function is the difference between two adjacent values which starts with Newton-Raphson method (NRM) and after the $9^{\text {th }}$ iteration the algorithm changes to modified Newton-Raphson method (MNRM) that converges to the solution in $7^{\text {th }}$ iterations. MAE measures the average magnitude of the errors in a set of predictions, without considering their direction. It's the average over the test sample of the absolute differences between prediction and actual observation where all individual differences have equal weight. Mean Absolute Error (MAE) is one of the most common metrics used to measure accuracy for continuous variables. According to this table $\varepsilon$ is Mean Absolute Error (MAE) or a tolerance criterion, which indicates the stopping criteria, the role of this parameter here in order to find the best approximate solution.

Table 1. The $\varepsilon$ for PV cell voltage using NRM and MNRM

\begin{tabular}{ccccc}
\hline Iterations & $V_{p v}$-NRM & $V_{p v}$-MNRM & $\varepsilon$-NRM & $\varepsilon$-MNRM \\
\hline 1 & 1 & 0.946728989 & 0.077576865 & 0.024305855 \\
2 & 0.971416861 & 0.929861208 & 0.048993727 & 0.007438073 \\
3 & 0.946732606 & 0.923246188 & 0.024309472 & 0.000823054 \\
4 & 0.929865706 & 0.922433925 & 0.007442571 & $1.07906 e^{-05}$ \\
5 & 0.923247893 & 0.922423136 & 0.000824759 & $1.79764 e^{-09}$ \\
6 & 0.922434 & 0.922423135 & $1.08655 e^{-05}$ & $1.11022 e^{-16}$ \\
7 & 0.922423136 & 0.922423135 & $1.9025 e^{-09}$ & 0.000000000 \\
8 & 0.922423135 & & $1.11022 e^{-16}$ & \\
9 & 0.922423135 & & 0.000000000 & \\
\hline
\end{tabular}


Figure 2 Presents the obtained solutions of the study result. The curves shows the pointwise error $\varepsilon$ of $x_{n}-x_{n-1}$ for problem Eq. 6 in case of number of iterations $\mathrm{N}=9$ and 7 using NRM and MNRM respectively. For the sake of comparison of our results with those obtained by applying these two different methods. The best error obtained by our two methods (NRM and MNRM), NRM was developed and this figure shows that our method MNRM is more accurate if compared with the results obtained in NRM.

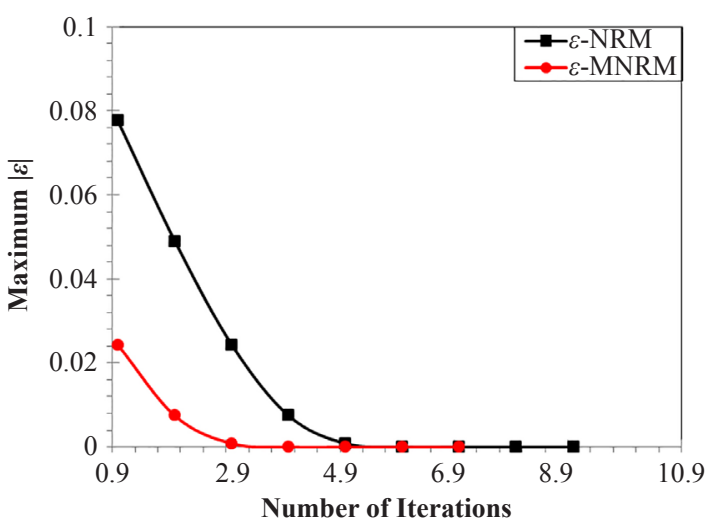

Figure 2. Obtained solutions of the study result

The obtained solution plots in the (no. of iteration)- $\varepsilon$-plane proves that the proposed method (MNRM) has small iterations compared with the other methods. Parallel to this feature, it is also noted that the proposed method (MNRM) has a behavior of the solution in the initial value $x_{0}=1$ has the smallest error tolerance compared with (NRM).

In Table 2 the methods NRM and MNRM with the comparison of the solution results are given and listed in the last column of this table when the load resistance $R=2$. From this table, the result of the iterative process, and the minimum stopping criterion $\varepsilon$, in which the objective function is the difference between two adjacent values which starts with Newton-Raphson method (NRM) and after the $9^{\text {th }}$ iteration the algorithm changes to modified Newton-Raphson method (MNRM) that converges to the solution in $7^{\text {th }}$ iterations.

Table 2. The $\varepsilon$ for PV cell voltage using NRM and MNRM

\begin{tabular}{ccccc}
\hline Iterations & $V_{p v^{-\mathrm{NRM}}}$ & $V_{p v^{-\mathrm{MNRM}}}$ & $\varepsilon$-NRM & $\varepsilon$-MNRM \\
\hline 1 & 1 & 0.945414147 & 0.082964618 & 0.028378765 \\
2 & 0.971030472 & 0.926823333 & 0.05399509 & 0.009787951 \\
3 & 0.945421967 & 0.918433095 & 0.028386584 & 0.001397713 \\
4 & 0.926834477 & 0.91706648 & 0.009799094 & $3.10972 e^{-05}$ \\
5 & 0.918438746 & 0.917035397 & 0.001403363 & $1.48468 e^{-08}$ \\
6 & 0.917066885 & 0.917035382 & $3.15024 e^{-05}$ & $1.0103 e^{-14}$ \\
7 & 0.917035399 & 0.917035382 & $1.61176 e^{-08}$ & 0.000000000 \\
8 & 0.917035382 & & $4.21885 e^{-15}$ & \\
9 & 0.917035382 & & 0.000000000 & \\
\hline
\end{tabular}

Figure 3 Presents the obtained solutions of the study result. The curves show the pointwise error $\varepsilon$ of $x_{n}-x_{n-1}$ for problem Eq. 6 in case of number of iterations $\mathrm{N}=9$ and 7 using NRM and MNRM respectively. For the sake of comparison of our results with those obtained by applying these two different methods. The best error obtained by our two methods (NRM and MNRM), NRM was developed and this figure shows that our method MNRM is more accurate if compared with the results obtained in NRM. 


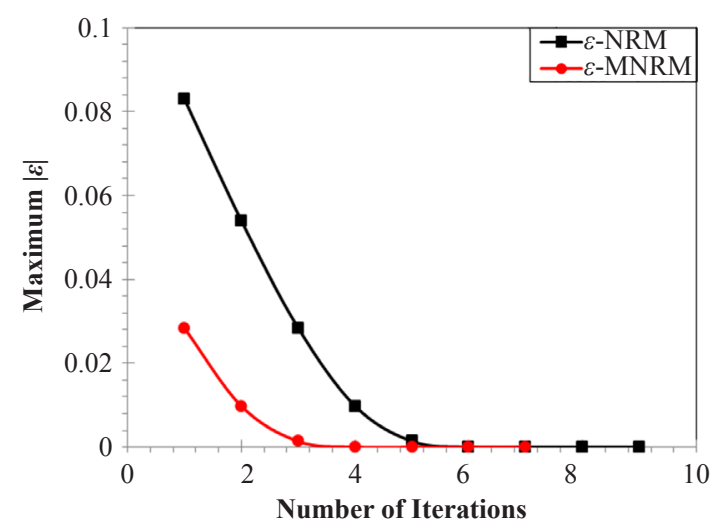

Figure 3. Obtained solutions of the study result

In Table 3 the methods NRM and MNRM with the comparison of the solution results are given and listed in the last column of this table when the load resistance $R=3$. From this table, the result of the iterative process, and the minimum stopping criterion $\varepsilon$, in which the objective function is the difference between two adjacent values which starts with Newton-Raphson method (NRM) and after the $9^{\text {th }}$ iteration the algorithm changes to modified Newton-Raphson method (MNRM) that converges to the solution in $7^{\text {th }}$ iterations.

Table 3. The $\varepsilon$ for PV cell voltage using NRM and MNRM

\begin{tabular}{ccccc}
\hline Iterations & $V_{p v}$-NRM & $V_{p v}$-MNRM & $\varepsilon$-NRM & $\varepsilon$-MNRM \\
\hline 1 & 1 & 0.944071555 & 0.089596626 & 0.033668181 \\
2 & 0.970643792 & 0.92357349 & 0.060240418 & 0.013170115 \\
3 & 0.944084232 & 0.91286348 & 0.033680858 & 0.002460106 \\
4 & 0.923594243 & 0.910499475 & 0.013190869 & $9.61007 e^{-05}$ \\
5 & 0.91287784 & 0.910403518 & 0.002474466 & $1.43895 e^{-07}$ \\
6 & 0.910501262 & 0.910403374 & $9.78883 e^{-05}$ & $4.26326 e^{-13}$ \\
7 & 0.910403531 & 0.910403374 & $1.57417 e^{-07}$ & 0.000000000 \\
8 & 0.910403374 & & $4.07563 e^{-13}$ & \\
9 & 0.910403374 & & 0.000000000 & \\
\hline
\end{tabular}

Figure 4 Presents the obtained solutions of the study result. The curves show the pointwise error $\varepsilon$ of $x_{n}-x_{n-1}$ for problem Eq. 6 in case of number of iterations $\mathrm{N}=9$ and 7 using NRM and MNRM respectively. For the sake of comparison of our results with those obtained by applying these two different methods. The best error obtained by our two methods (NRM and MNRM), NRM was developed and this figure shows that our method MNRM is more accurate if compared with the results obtained in NRM.

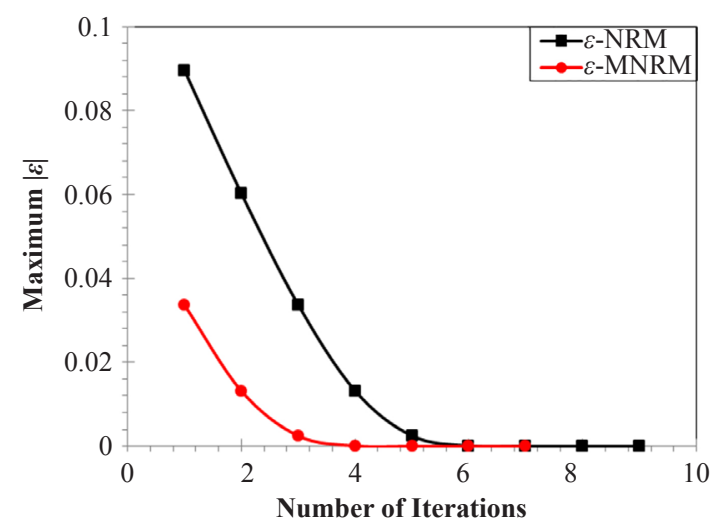

Figure 4. Obtained solutions of the study result 
In Table 4 the methods NRM and MNRM with the comparison of the solution results are given and listed in the last column of this table when the load resistance $R=4$. From this table, the result of the iterative process, and the minimum stopping criterion $\varepsilon$, in which the objective function is the difference between two adjacent values which starts with Newton-Raphson method (NRM) and after the $9^{\text {th }}$ iteration the algorithm changes to modified Newton-Raphson method (MNRM) that converges to the solution in $7^{\text {th }}$ iterations.

Table 4. The $\varepsilon$ for PV cell voltage using NRM and MNRM

\begin{tabular}{ccccc}
\hline Iterations & $V_{p v}$-NRM & $V_{p v^{-M N R M}}$ & $\varepsilon$-NRM & $\varepsilon$-MNRM \\
\hline 1 & 1 & 0.942700461 & 0.098259398 & 0.040959859 \\
2 & 0.970256822 & 0.920088564 & 0.06851622 & 0.018347962 \\
3 & 0.94271872 & 0.906313217 & 0.040978118 & 0.004572615 \\
4 & 0.920123009 & 0.902069876 & 0.018382407 & 0.000329274 \\
5 & 0.906346494 & 0.90174234 & 0.004605892 & $1.7381 e^{-06}$ \\
6 & 0.902077706 & 0.901740602 & 0.000337104 & $3.61988 e^{-12}$ \\
7 & 0.901742503 & 0.901740602 & $1.90088 e^{-06}$ & 0.000000000 \\
8 & 0.901740602 & & $6.06911 e^{-11}$ & \\
9 & 0.901740602 & & 0.000000000 & \\
\hline
\end{tabular}

Figure 5 Presents the obtained solutions of the study result. The curves show the pointwise error $\varepsilon$ of $x_{n}-x_{n}-1$ for problem Eq. 6 in case of number of iterations $\mathrm{N}=9$ and 7 using NRM and MNRM respectively. For the sake of comparison of our results with those obtained by applying these two different methods. The best error obtained by our two methods (NRM and MNRM), NRM was developed and this figure shows that our method MNRM is more accurate if compared with the results obtained in NRM.

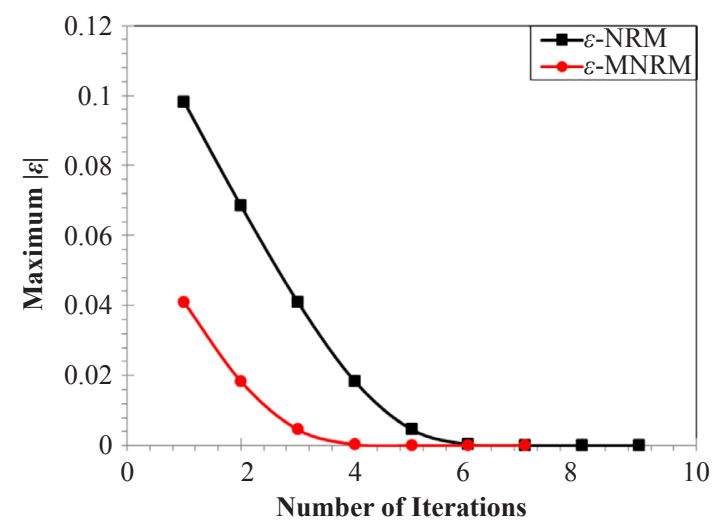

Figure 5. Obtained solutions of the study result

In Table 5 the methods NRM and MNRM with the comparison of the solution results are given and listed in the last column of this table when the load resistance $R=5$. From this table, the result of the iterative process, and the minimum stopping criterion $\varepsilon$, in which the objective function is the difference between two adjacent values which starts with Newton-Raphson method (NRM) and after the $10^{\text {th }}$ iteration the algorithm changes to modified Newton-Raphson method (MNRM) that converges to the solution in $8^{\text {th }}$ iterations. 
Table 5. The $\varepsilon$ for PV cell voltage using NRM and MNRM

\begin{tabular}{ccccc}
\hline Iterations & $V_{p v^{-N R M}}$ & $V_{p v}$-MNRM & $\varepsilon$-NRM & $\varepsilon$-MNRM \\
\hline 1 & 1 & 0.941300083 & 0.110907285 & 0.052207368 \\
2 & 0.96986956 & 0.916342096 & 0.080776845 & 0.027249381 \\
3 & 0.941324731 & 0.898461163 & 0.052232016 & 0.009368448 \\
4 & 0.916395843 & 0.890439476 & 0.027303128 & 0.001346762 \\
5 & 0.898535645 & 0.889122977 & 0.00944293 & $3.02626 e^{-05}$ \\
6 & 0.890477009 & 0.889092724 & 0.001384294 & $9.66595 e^{-09}$ \\
7 & 0.889125763 & 0.889092715 & $3.30483 e^{-05}$ & $1.22791 e^{-13}$ \\
8 & 0.889092734 & 0.889092715 & $1.91907 e^{-08}$ & 0.000000000 \\
9 & 0.889092715 & & 0.000000000 & \\
10 & 0.889092715 & & 0.000000000 & \\
\hline
\end{tabular}

Figure 6 Presents the obtained solutions of the study result. The curves show the pointwise error $\varepsilon$ of $x_{n}-x_{n}-1$ for problem Eq. 6 in case of number of iterations $\mathrm{N}=9$ and 7 using NRM and MNRM respectively. For the sake of comparison of our results with those obtained by applying these two different methods. The best error obtained by our two methods (NRM and MNRM), NRM was developed and this figure shows that our method MNRM is more accurate if compared with the results obtained in NRM.

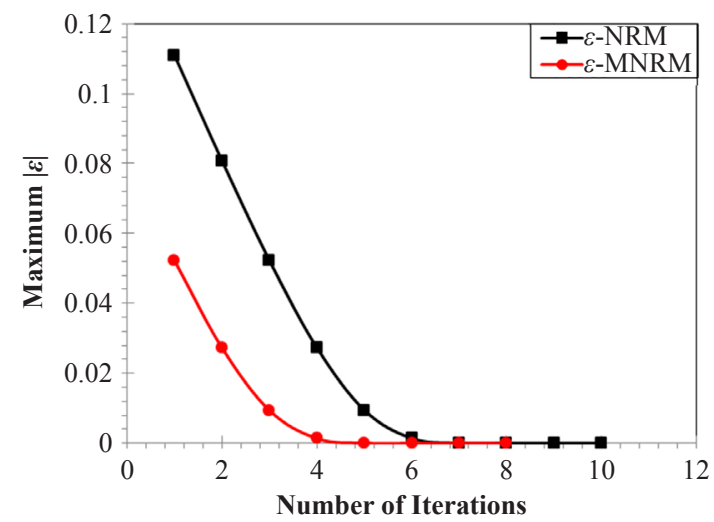

Figure 6. Obtained solutions of the study result

Results of tables 1 to 5 show that the suggested method (MNRM) has low error after relatively view iterations are computed, and this in turn is demonstrating their efficiency.

\section{Conclusion}

This research, gives two numerical solutions of a mathematical model for single-diode photovoltaic cells. The purpose of the comparison between the two methods NRM and MNRM is to obtain the results with the least number of iterations, which proves that the mathematical method with the least number of iterations is better and faster. The results proved with NRM, nine iterations were used, while using the MNRM, seven iterations were used.

\section{References}

[1] Delphi M, Shihab S. State parametrization basic spline functional for trajectory optimization. The Journal of Nature Life and Applied Sciences. 2019; 3(4): 110-119.

[2] Delphi M, Shihab S. Modified basic spline multiscaling and wavelets algorithms for optimal control problems. Baghdad: University of Technology; 2019.

[3] Delphi M, Shihab S. Operational matrix basic spline wavelets of derivative for linear optimal control problem. Electronics Science Technology and Application. 2019; 6(2): 18-24. 
[4] Dephi M, Shihab S. Modified iterative algorithm for solving optimal control problems. Open Science Journal of Statistics and Application. 2019; 6(2): 20.

[5] Shihab S, Dephi M. Direct iterative algorithm for solving optimal control problems using b-spline polynomials. Emirates Journal for Engineering Research. 2019; 24(4): 2.

[6] Rasheed, M., Sarhan, M. A. Solve and implement the main equations of photovoltaic cell parameters using visual Studio Program. Insight-Mathematics. 2019; 1(1): 18-26.

[7] Tahir, F. S., Rasheed, M. S., Hameed, I. A. Analysis the performance of silicon solar cell parameters with the ambient temperature using fuzzy logic. Journal of the College of Basic Education. 2012; 18(75): 173-183.

[8] Rasheed, M., Sarhan, M. A. Characteristics of solar cell outdoor measurements using fuzzy logic method. InsightMathematics. 2019; 1(1): 1-8.

[9] Rasheed, M. S. Investigation of solar cell factors using fuzzy set technique. Insight-Electronic. 2019; 1(1): 17-23.

[10] Muna Muzahim Abbas, Rasheed, M. Solid state reaction synthesis and characterization of aluminum doped titanium dioxide nanomaterials. Journal of Southwest Jiaotong University. 2020; 55(2): 1-10. 